\title{
New Ultrasonic Techniques for Detecting and Quantifying Railway Wheel-Flats
}

\author{
Jose Brizuela ${ }^{1}$, Carlos Fritsch $^{1}$ and Alberto Ibáñez ${ }^{2}$ \\ ${ }^{1}$ Consejo Superior de Investigaciones Científicas (CSIC) \\ ${ }^{2}$ Centro de Acústica Aplicada y Evaluación No Destructiva - CAEND (CSIC-UPM) \\ Spain
}

\section{Introduction}

The railway transport braking processes are likely to form surface defects on the tread if the wheel locks up and slides along the rail. This action can be produced by a defective, frozen or incorrectly tuned brake, as well as by a low rail-wheel adhesion caused by environmental conditions (rain, snow, leaves, etc.). The abrasive effect of skidding causes a high wear on the rolling surface (a wheel-flat), with lengths ranging typically from 20 to over $100 \mathrm{~mm}$.

The rise in temperature caused by abrasion followed by a fast cooling may lead to the formation of brittle martensite beneath the wheel-flat. This can be associated to the beginning of further flaws like cracks and spalls with the loss of relatively large pieces of tread material. When the wheel rolls over a flat, high impact forces are developed and may cause a rapid deterioration of both, rolling and fixed railway structures. Moreover, the incidence of hot bearings, broken wheels and rail fractures are coincidental with the number of wheel-flats and out-of-round wheels (Kumagai et al., 1991; Snyder \& Stone, 2003; Vyas \& Gupta, 2006; Zakharov \& Goryacheva, 2005).

Besides, when a critical speed is reached, a loss of rail-wheel contact occurs which produces high-levels of noise and vibrations that also affect passengers comfort. Finally in the worst-case scenario, surface defects on treads can cause a derailment (Wu \& Thompson, 2002; Zerbst et al., 2005).

Therefore, there is a great interest in finding methods for an early detection and evaluation of surface defects without dismantling wheelsets due to their complex assembly (Pohl et al., 2004). Ideally, in order to reduce time and costs, inspection systems should be placed at the end of train-wash stations or at the entrance of maintenance shops, where trains pass frequently at low speed.

Nowadays, many automatic and on-line wheel tread surface defect detection systems have also been developed for the railway industry. Most of them can be classified into one of the following categories:

Measurement of the impact forces in an instrumented rail: the Wheel Impact Load Detector (WILD) developed by Salient Systems, Inc. (2010) is the most popular system to detect wheel-flats; it consists of a large number of strain gauges mounted in the rail web, which 
are used to quantify the force applied to the rail through a mathematical relationship between the applied load and the deflection of the foot of the rail. As a result, the wheels health and the safe train operations can be ensured by monitoring these impact forces (Stratman et al., 2007). In other cases, accelerometers are used instead (Belotti et al., 2006; Madejski, 2006). These techniques analyse the impacts produced by flats or other kind of flaws, but give no indications about their size.

Wheel radius variations measurement: in this case, the flange is considered perfectly round and wear-free, being used as a reference to estimate the variations in the wheel radius. Mechanical (Feng et al., 2000; He et al., 2005) and optical (Gutauskas, 1992) systems have been designed based on this idea. Nevertheless, the hostile railway environment involves some disadvantages for these methods, both are sensitive to vibrations and their resolution is limited. Furthermore, small irregularities or adherences in the flange will lead to false indications.

Ultrasonic flaw detection and measurement: the Non Destructive Testing (NDT) techniques by ultrasound are often used for offline wheel examination; most of them require complex installations and/or machineries (Kappes et al., 2000). However, recent designs have been developed for online wheel tread inspection using ultrasonic methods. Such systems consist in sending an ultrasound pulse over the rolling surface to detect echoes produced by cracks. The interrogating Rayleigh wave is generated by a transducer (EMAT or piezoelectric) which is fired when the wheel passes over it; the same or other sensor can receive reflections originated on flaws (Fan \& Jia, 2008; Ibáñez et al., 2005; Salzburger et al., 2008). Unfortunately, wheel-flats usually have smooth edges that do not generate echoes, so it is very difficult to detect them by these techniques. Moreover, acoustic coupling between transducers and wheels frequently give rise to unreliable measurements due to variability problems.

The aim of this chapter is to present an innovative ultrasound technique designed to detect and quantify wheel-flats that have been formed on the railway wheel tread. An extended theoretical framework supports the proposed method. It can be applied to trains moving at low speed (10-15 Km/h typical) and allows all the wheelsets mounted in a train to be inspected within a few seconds.

\section{Alternative methodology}

The proposed method differs from other conventional ultrasonic flaw detection approaches, which are based on the reflectivity of static flaws. In this case, surface waves are sent over a measuring rail instead of the rolling surface to interrogate the rail-wheel contact point position. Wheel-flats are then detected and sized by analysing the kinematic of the wheel-rail contact point echo.

The proposed technique comes up with many other advantages for railway industry, e.g., no moving parts are involved in the measuring system; the set of transducer and measuring-rail is invariant, so it can be fully characterized once; results are independent of wheel wear degree, no exploring pulses along rolling surface are sent; finally, an optimum ultrasonic coupling between transducer and rail can be achieved.

Following this methodology two alternatives have been considered to determine wheel-flats: the first one is based on Doppler techniques which are suitable to detect velocity changes of a moving reflector (in this case the rail-wheel contact point). The second consist in measuring 


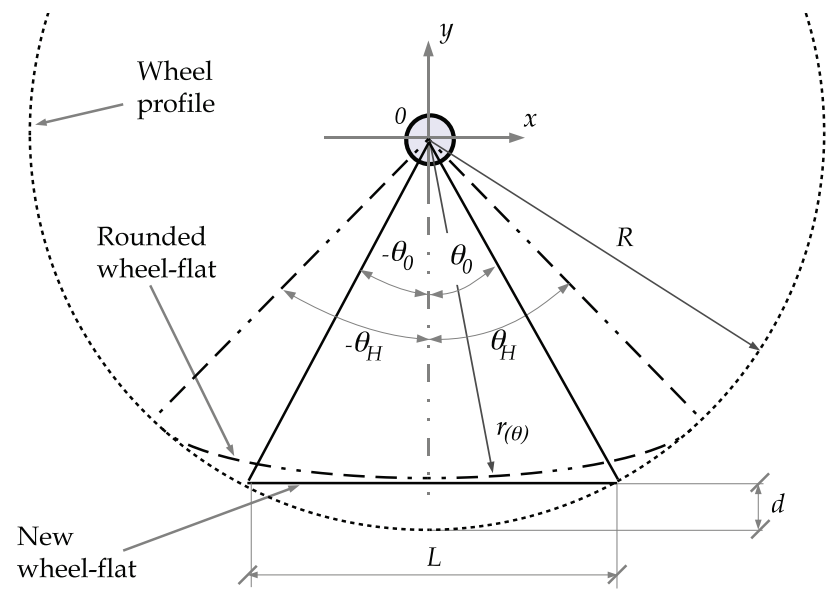

Fig. 1. Large-scale representation of a new wheel-flat and its wear stage.

the round trip time of flight (RTOF) of the echo produced by the rail-wheel contact point. The RTOF value variations allow detecting and quantifying wheel-flats with enough resolution.

\subsection{Theoretical background}

A wheel-flat of recent formation (new or fresh wheel-flat stage) can be represented as a circumference chord defined by two parameters: its length $L$ and the loss of material $d$. The wheel-flat edges are singular points, where the curvature changes from the nominal wheel radius $R$ to $\infty$ in the angular interval $\left[-\theta_{0}, \theta_{0}\right]$. The geometry shown in Fig. 1 lets to obtain the length $L$ as a function of $d$ as follows:

$$
L=2 \sqrt{2 R d-d^{2}} \approx \sqrt{8 R d}
$$

Nevertheless, as the wheel keeps in motion, the wheel-flat edges become worn progressively by plastic deformation (partially rounded wheel-flat stage). Finally, the irregularity profile eventually disappears due to the successive impacts over the rail (degenerated or rounded wheel-flat). At this stage, the wheel-flat becomes a continuous curve of length greater than $L$. The wheel wear may cause a further increase of the wheel-flat length but, since this process is rather slow and uniform, so the loss of material $d$ remains unchanged until it is removed by a turning machine (Baeza et al., 2006). However, if the rounded wheel-flat length increases above four times from the original size, the defect makes the wheel be out-of-round (Snyder \& Stone, 2003).

The wheel tread having a wheel-flat can be described by a function $r(\theta)<R$ in the irregularity and $r(\theta)=R$ outside of the angular interval which defines the defect. For degenerated wheel-flats, this function is continuous with no singular points, so that $\dot{r}(\theta)$ denotes a smooth unbroken curve as well. Although not strictly required, it will be also assumed for clearness a symmetric wheel-flat profile around the interval center located at the point of maximum loss of radius: $d=R-R_{\min }$ (Fig. 1 ). 


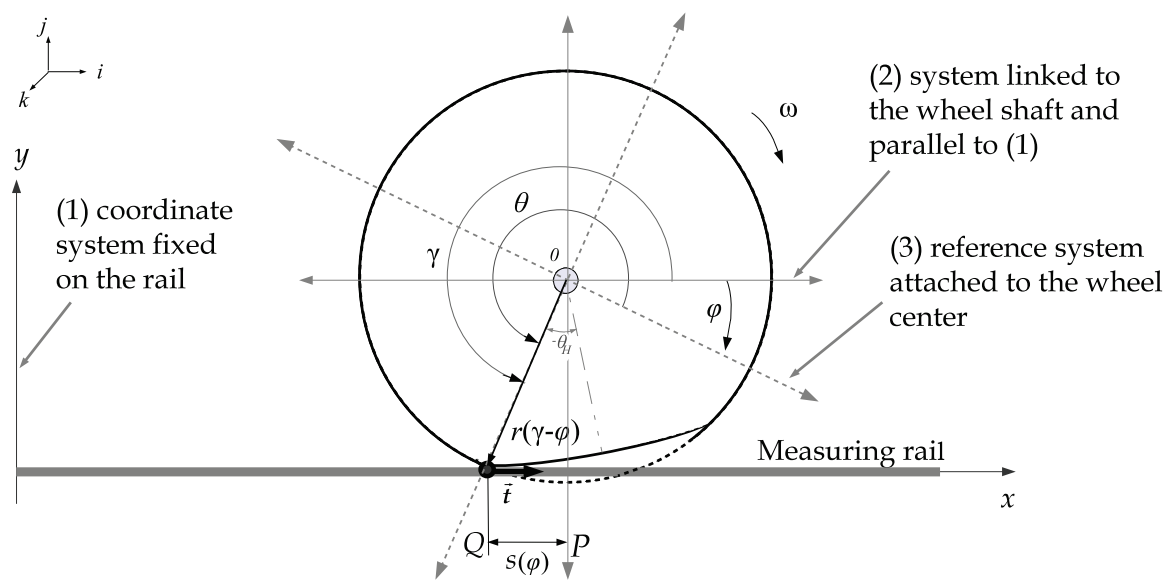

Fig. 2. Scheme used to determine the wheel-flat loss of material $d$. The wheel moves towards $+x$ direction on the reference system (1). Pay attention that $\varphi<0$ and the displacement $s<0$. It is assumed that effects such as creep, spin, and slip, are ignored, so the contact is not lost in the time domain. The contact point $Q$ is defined in system (2) by its polar coordinates $(r(\theta), \theta)=(r(\gamma-\varphi), \gamma-\varphi)$, while in (3) they are $(q(\gamma, \varphi), \gamma)$. The rotation angle $\varphi$ at each instant represents the difference between fixed and mobile systems, both mounted on the wheel center.

\subsubsection{Rail-wheel contact point kinematic}

The wheel rotation around its contact point $Q$ can be described by following the scheme in Fig. 2, where can be find three reference systems:

1) A coordinate system fixed on the measuring rail.

2) A system linked to the wheel shaft and parallel to the system 1.

3) A coordinate system attached to the wheel center in motion with vector $\vec{Q}$.

Note that, the rail is always tangent to the wheel at the contact point. When the wheel rolls over a perfectly rounded region the wheel center projection $P$ on the rail coincides with the wheel-rail contact point $Q$ (that means $P=Q$ ) since, in a circunference, radius and unitary tangent vector $(\vec{t})$ at $Q$ are perpendicular. However, when the wheel rolls over its non circular region $\left[-\theta_{H}, \theta_{H}\right]$ it rotates an angle $\varphi$, so vectors $\vec{Q}$ and $\vec{t}$ are not orthogonal. Moreover, the contact point and the projection are moved apart in a distance $s$ which is a function of $\varphi$, reflecting an advance or delay of $Q$ in relation to $P$. In other words:

a) when $-\theta_{H}<\varphi \leq 0$, $P$ leads $Q$ and $s \leq 0$;

b) for $0<\varphi<\theta_{H}, P$ lags $Q$ and $s>0$.

Fig. 2 shows the situation when $\varphi<0$, so the contact point is behind the wheel center projection. On the other hand, each point of the wheel tread is described by $\vec{r}(\theta)=(r(\theta), \theta)$ on reference system 2, while on system 3 the coordinates are: $(r(\theta), \theta)=(q(\gamma, \varphi), \gamma)$. Then, the transition from one system to another is given by:

$$
q(\gamma, \varphi)=r(\gamma-\varphi)
$$


and

$$
\frac{\partial(q(\gamma, \varphi))}{\partial \gamma}=\frac{\partial(r(\gamma-\varphi))}{\partial \gamma}=\dot{r}(\gamma-\varphi)
$$

Assuming that contact is not lost while the wheel is moving and the train speed $v$ is constant over the measuring rail. So, the rail-wheel contact point is defined for each rotated angle $\varphi$ as $Q=\left(q\left(\gamma_{Q}, \varphi\right), \gamma_{Q}\right)$, such that its ordinate value $q(\gamma, \varphi) \sin (\gamma)$ is minimal on $\gamma_{Q}$, that is:

$$
\left.\frac{\partial(q(\gamma, \varphi) \sin (\gamma))}{\partial \gamma}\right|_{\gamma=\gamma_{Q}}=\left.\frac{\partial(q(\gamma, \varphi))}{\partial \gamma}\right|_{\gamma=\gamma_{Q}} \sin \left(\gamma_{Q}\right)+q\left(\gamma_{Q}, \varphi\right) \cos \left(\gamma_{Q}\right)=0
$$

Replacing eqs. (2) and (3) on (4), it is possible to figure out the tangent value at $Q$, which is:

$$
\tan \left(\gamma_{Q}\right)=-\frac{r\left(\gamma_{Q}-\varphi\right)}{\dot{r}\left(\gamma_{Q}-\varphi\right)}
$$

The contact point $Q$ belongs to the instantaneous axis of rotation, which remains steady for each $\varphi$ value, and the wheel center movement can be described, at all time, as a pure rotation around $Q$. Additionally, as the angular velocity $\omega$ is an invariant and independent of any reference systems used, the velocity vector is defined by:

$$
\vec{v}=-\vec{\omega} \times \vec{Q}=-\vec{\omega} \times \vec{r}\left(\gamma_{Q}-\varphi\right)
$$

where

$$
\begin{aligned}
\vec{\omega} & =\omega \vec{k} \\
\vec{r}\left(\gamma_{Q}-\varphi\right) & =\left(q\left(\gamma_{Q}, \varphi\right) \sin \left(\gamma_{Q}\right)\right) \vec{j}+\left(q\left(\gamma_{Q}, \varphi\right) \cos \left(\gamma_{Q}\right)\right) \vec{i}
\end{aligned}
$$

Solving the cross product (6), it yields to:

$$
\vec{v}=\left|\begin{array}{ccc}
\vec{i} & \vec{j} & \vec{k} \\
0 & 0 & \omega \\
q\left(\gamma_{Q}, \varphi\right) \cos \gamma_{Q} & q\left(\gamma_{Q}, \varphi\right) \sin \gamma_{Q} & 0
\end{array}\right|=\omega q\left(\gamma_{Q}, \varphi\right) \sin \left(\gamma_{Q}\right) \vec{i}-\omega q\left(\gamma_{Q}, \varphi\right) \cos \left(\gamma_{Q}\right) \vec{j}
$$

As a result, there are two velocity components on (9). The first on $\vec{i}$-direction, means the train speed. By taking into account (2):

$$
v=\omega q\left(\gamma_{Q}, \varphi\right) \sin \left(\gamma_{Q}\right)=\omega r\left(\gamma_{Q}-\varphi\right) \sin \left(\gamma_{Q}\right)
$$

On the other hand, regarding eqs. (5) and (10), the velocity component in the $\vec{j}$-direction is:

$$
\overrightarrow{v_{y}}=-\omega q\left(\gamma_{Q}, \varphi\right) \cos \left(\gamma_{Q}\right)=\omega \dot{r}\left(\gamma_{Q}-\varphi\right) \sin \left(\gamma_{Q}\right)=-\frac{v}{\tan \left(\gamma_{Q}\right)}
$$

As (11) expresses, for a perfect rounded wheel, the contact point $Q$ will be located at $3 \pi / 2$, so $\overrightarrow{v_{y}}$ will be null. Note as well on Fig. 2, that the displacement distance between the contact point $Q$ and the wheel center projection $P$ denoted by $s(\varphi)$ is the $x$-coordinate on the reference system 2 . That is:

$$
s(\varphi)=-r\left(\gamma_{Q}-\varphi\right) \cos \left(\gamma_{Q}\right)=-q(\gamma, \varphi) \cos \left(\gamma_{Q}\right)
$$




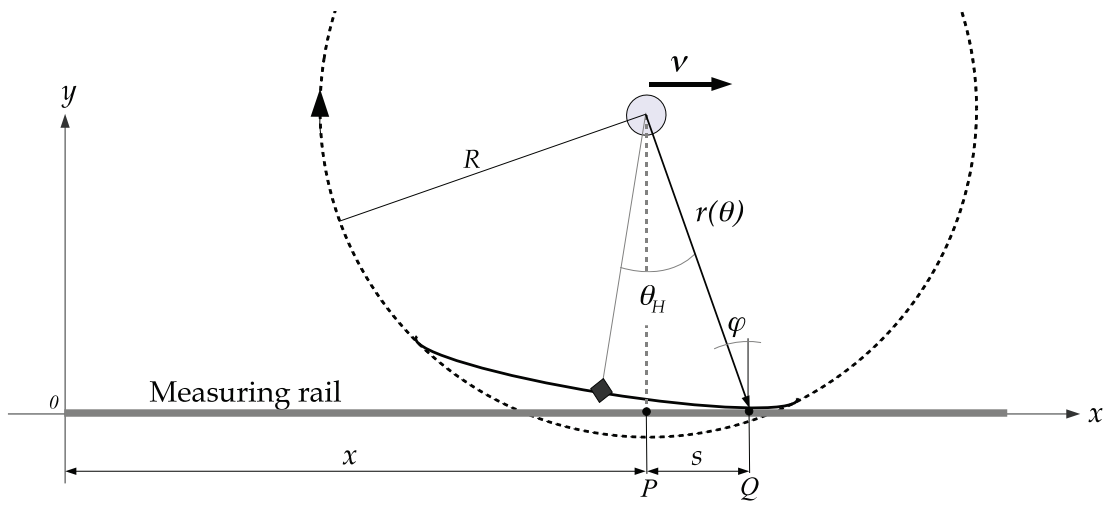

Fig. 3. Position of points $P$ and $Q$ on the reference system fixed to the rail, when the wheel passes over an irregularity.

Therefore, the wheel center vertical movement can be related with the displacement $s(\varphi)$ by replacing (12) on (11):

$$
\overrightarrow{v_{y}}=\omega s(\varphi)
$$

Thus, the wheel center vertical movement is zero for perfectly rounded wheels. However over a wheel-flat irregularity, the wheel center goes down a distance equal to the maximum loss of material $d$. Afterwards, it starts to rise again up to reach the radius nominal value just when the contact point comes out of the irregularity. As a result, the total wheel center displacement is $2 d$ from the time instant $t_{1}$ to $t_{2}$, when the wheel rolls over the irregularity:

$$
2 d=\int_{t_{1}}^{t_{2}}\left|v_{y}\right| \mathrm{d} t=\int_{t_{1}}^{t_{2}}|\omega s(\varphi)| \mathrm{d} t=\int_{-\varphi_{H}}^{\varphi_{H}}|s(\varphi)| \mathrm{d} \varphi
$$

where the limits of integration $-\varphi_{H}$ and $\varphi_{H}$ correspond to the angular range that determines the irregularity. Hence, (14) gives the parameter $d$ as a function of displacements $s(\varphi)$ which can be measured; then the original wheel-flat length is obtained by (1). On the other hand, as $s(\varphi)$ is a continuous function even if singular points are present, (14) is valid for any kind of wheel-flats (new, partially rounded or degenerated). This property can be generalized as:

"For small irregularities, whatever is the wheel-flat wear degree from the original one, the area below $|s(\varphi)|$ is two times the loss of material $d^{\prime \prime}$.

As well, since $s(\varphi)=0$ in the round part of the wheel $\left(|\varphi|>\varphi_{H}\right)$, the limits of integration may be extended to any angle $\varphi_{A} \geq \varphi$ for single or isolated wheel-flats cases. Using an auxiliary parameter $\alpha$ in order to cover a full wheel revolution (14) can be written as:

$$
d(\alpha)=\int_{\alpha}^{\alpha+\varphi_{A}} s(\varphi) \mathrm{d} \varphi \quad \text { where } \quad 0 \leq \alpha \leq 2 \pi-\varphi_{A} \quad \text { and } \quad \varphi_{A} \geq \varphi
$$

\subsubsection{Rail-wheel contact point velocity}

When the wheel moves over the rail the instantaneous position of the wheel center projection is given by $x$, while the contact point $Q$ is $x+s$. Note that the distance $x$ is now measured 


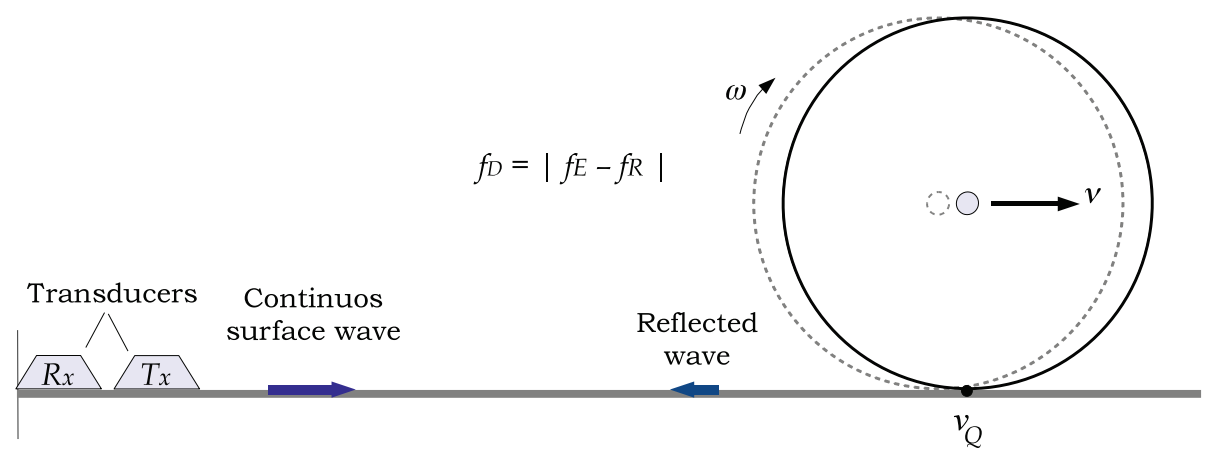

Fig. 4. Arrangement used for wheel-flat detection by Doppler effect.

from the origin located at reference system fixed to the rail (Fig. 3). The time derivative of the $Q$ position gives the wheel-rail contact velocity as:

$$
v_{Q}=\frac{\mathrm{d} x}{\mathrm{~d} t}+\frac{\mathrm{d} s}{\mathrm{~d} t}=v+\frac{\mathrm{d} s}{\mathrm{~d} t}=v+\omega \frac{\mathrm{d} s}{\mathrm{~d} \varphi}
$$

where $v$ is the train speed. As the wheel rolls over a perfectly rounded region, $s=0$ and $v_{Q}=$ $v$. However when the contact point reaches an irregularity, $s$ takes negative values because the point $Q$ is behind the wheel center projection $P$ and $v_{Q} \leq v$. On the other hand, when $Q$ is ahead of $P, s$ becomes positive and $v_{Q} \geq v$. Therefore, the presence of an irregularity can be determined by measuring variations in the contact point velocity; or what is the same, the time variations of $s$, or $s$ in relation to $\varphi$, since $\mathrm{d} \varphi=\omega \mathrm{d} t$.

\section{Measuring techniques}

Taking into account the arrangement shown on Fig. 3, the rail can be used as the physical support for Rayleigh waves in order to determine velocity changes while the rail-wheel contact point is in motion.

A conventional ultrasound transducer mounted on a plastic wedge, which has a propagation velocity $c_{w}$, can be used to generate Rayleigh waves by properly adjusting the incidence angle $\beta$ of the emitted signal (Bray et al., 1973). Following the Snell's law for refraction at $90^{\circ}$ :

$$
\sin (\beta)=\frac{c_{w}}{c}
$$

By this way, ultrasonic waves propagate over the measuring rail, eventually producing an echo when it arrives at the rail-wheel contact point. Two alternatives to perform the measurement will be described as follows.

\subsection{Wheel-flat detection using Doppler effect}

In this case, monochromatic surface waves of frequency $f_{E}$ generated by an emitter transducer are propagated over the measuring rail. Another sensor receives the weak echo signal produced by the wheel-rail contact. The emitting transducer is placed ahead of the receiver to avoid reflections; both are fixed to the rail to achieve a better acoustic coupling (Fig. 4).

The received frequency $f_{R}$, once amplified, is compared with the emitted $f_{E}$ by a quadrature 
Transducer

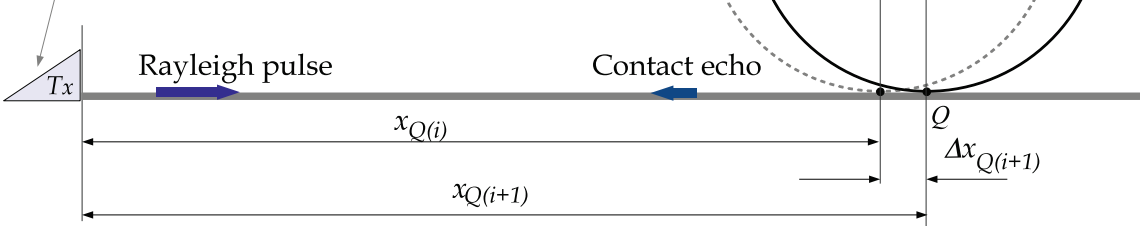

Fig. 5. Arrangement used for measuring the round trip time of flight (RTOF).

demodulator for recovering the Doppler frequency $f_{D}=\left|f_{E}-f_{R}\right|$. This frequency shift is proportional to the rail-wheel contact point velocity $v_{Q}$ by:

$$
f_{D}=2 \frac{v_{Q}}{c} f_{E}
$$

where $c$ is the propagation velocity for Rayleigh waves. Then by using (16), it is possible to reach the following expression:

$$
f_{D}=\frac{2 v}{c} f_{E}+\frac{2 f_{E}}{c} \frac{\mathrm{d} s}{\mathrm{~d} t}
$$

The first term on (19) represents the nominal Doppler shift which is considered constant and it is proportional to the train speed. When the wheel rolls over a perfectly rounded region, the second term is zero $(s=0)$. However if the wheel passes over a wheel-flat, the displacement $s$ varies over the time, shifting the nominal Doppler frequency. Then:

$$
\frac{\mathrm{d} s}{\mathrm{~d} t}=\frac{c}{2 f_{E}} f_{D}-v
$$

Moreover, (20) can be easily implemented for detecting flaws in a continuous way as:

$$
s(t)=\left(\frac{c}{2 f_{E}} f_{D}-v\right) t
$$

since $c, f_{E}$ are design data, and $f_{D}, v$ are the measurements taken.

The practical implementation of (20) is restricted by the time-frequency uncertainty conflict which makes the resolution be reduced. This limitation must be addressed through compromise solutions to get reliable flaw indications. Nevertheless, wheel-flats longer than $30 \mathrm{~mm}$ can be detected although sizing is difficult. The method also provides a time-frequency analysis that gives a quick reference about the wheel tread state (Brizuela, Ibáñez, Nevado \& Fritsch, 2010).

\subsection{Wheel-flat detection by measuring the round trip time of flight (RTOF):}

This alternative uses only one transducer located at one end of the rail (Fig. 5). The sensor generates pulses of Rayleigh waves with a period $T_{P R F}$ and receives the echo produced by the 
moving rail-wheel contact point. The rail-wheel contact position obtained from measuring the $\operatorname{RTOF}\left(T_{Q(i)}\right)$ of a pulse $i$ is:

$$
x_{Q(i)}=\frac{c T_{Q(i)}}{2}
$$

where $c$ also means the propagation velocity for Rayleigh waves. Assuming that the train moves at a constant speed $v$; the wheel center projection over the rail for the same pulse $i$ is:

$$
x_{P(i)}=i T_{P R F} v
$$

Therefore, the relative displacement between $P$ and $Q$ can be figured out as follows:

$$
s(i)=x_{Q(i)}-x_{P(i)}=\frac{c T_{Q(i)}}{2}-i T_{P R F} v
$$

On the other hand, a sampled system can approach the integration indicated on (15) as:

$$
d_{k}(M)=\frac{v T_{P R F}}{R} \sum_{i=k}^{k+M} s(i)
$$

where the differential on (15) has been replaced by $\Delta \varphi=\omega \Delta t=v T_{P R F} / R$ and $M$ is the discrete version of the angle $\varphi_{A}$. The sum given by (25) is extended over $M$ samples of $s(i)$ which is obtained from (24). Consequently, $M$ should be chosen to cover at least half the largest irregularity. Since the sampling period of $s(i)$ is $T_{P R F}$ and $L_{\max }$ is the length of the largest wheel-flat of interest,

$$
M \geq \frac{L_{\max }}{2 v T_{P R F}}
$$

The measuring process (25) is carried out as a convolution of a rectangular unity window of $M$ samples with the values of $s(i)$. This way $M$ measurements of $d$ are taken: the sequence $d_{k}(M)$ represents the value of the loss of material $d$, which is estimated by convolving the $k$ with a window of size $M$. Since the window must be wider than the irregularity, the peak value of this sequence corresponds to the best estimation of $d$ for isolated wheel-flats. Fig. 6 shows graphically the measuring process. For a given value of $M$ samples, the resulting sequence $d_{k}(M)$ has two peaks, one negative $d_{N}$ followed by another positive $d_{P}$, which correspond to the lead-lag of $P$ respect to $Q$ on the signal displacement $s$. Their absolute values scaled by the factor $v T_{P R F} / R$, make the result be equal to $d$. However in real applications where noise is present, $d$ can be much better estimated by the peak-to-peak average:

$$
d_{E}(M)=\frac{v T_{P R F}}{R} \frac{\left|d_{P}(M)\right|+\left|d_{N}(M)\right|}{2}
$$

Once the estimated value $d_{E}$ has been found, the length corresponding to the original wheel-flat $L_{E}$ can be obtained by application of (1).

This methodology also provides a suitable possibility of estimating the train speed required by (27). The contact point velocity is $v_{Q}=v$ while the wheel rolls over a defect-free region, otherwise during an irregularity $v_{Q} \neq v$. Nevertheless, the average speed along the wheel-flat is $\hat{v}_{Q}=v$. Consequently, by calculating the contact point mean-speed for a long enough time interval the train speed is obtained with a good accuracy. 


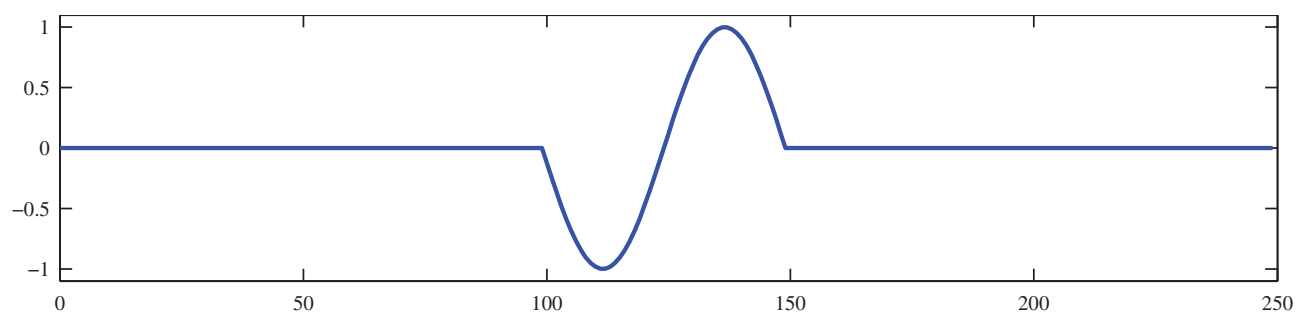

(a) Displacement signal $s$

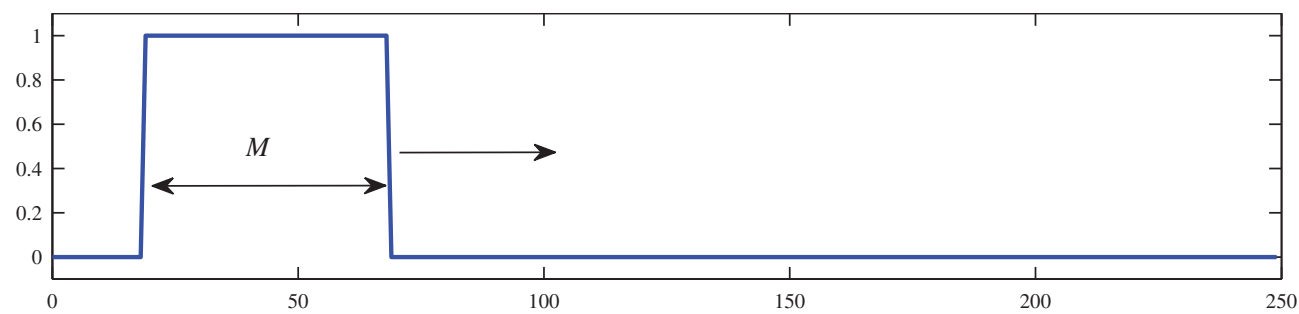

(b) Rectangular unity window $\mathbf{w}$

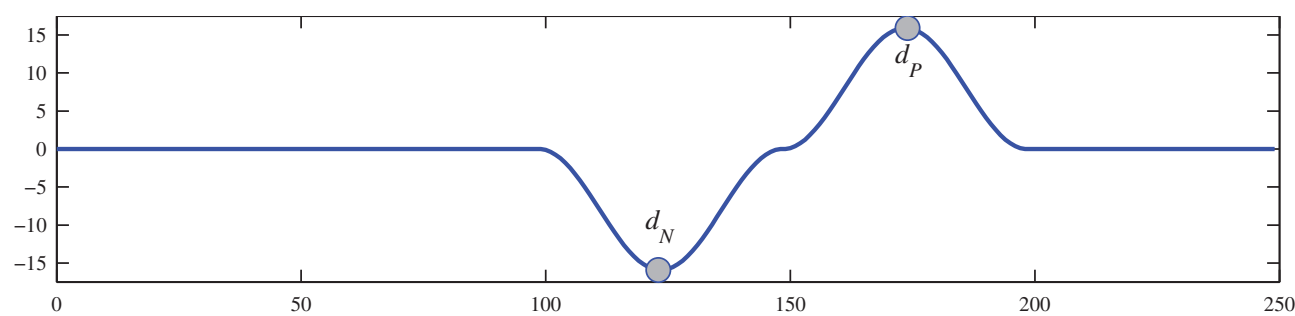

(c) Resulting sequence $d_{k}=s * \mathbf{w}$.

Fig. 6. Measuring process carried out as a convolution of $s$ with an unit window of $M$ samples.

The contact point moves a distance $\Delta x_{Q(i)}$ between two consecutive pulses. The $x_{Q(i)}$ position is measured at a time:

$$
t_{(i)}=i T_{P R F}+\frac{T_{Q(i)}}{2}
$$

in the following interrogation pulse, the $Q$ position is sampled at:

$$
t_{(i+1)}=(i+1) T_{P R F}+\frac{T_{Q(i+1)}}{2}
$$

The elapsed time between measures (28) and (29) is:

$$
\Delta t_{(i)}=T_{P R F}+\frac{\Delta T_{Q(i)}}{2}
$$

The $Q$ movement is:

$$
\Delta x_{Q(i)}=v_{Q(i)} \Delta t_{(i)}
$$


At the same time, the ultrasound pulse has also to cover the distance advanced by the contact point, thus:

$$
\Delta T_{Q(i)}=2 \frac{\Delta x_{Q(i)}}{c}
$$

where the constant $c$ is the propagation velocity for Rayleigh waves on the rail. By replacing (31) on (32) and then combining on (30), it yields:

$$
\Delta T_{Q(i)}\left(\frac{c-v_{Q(i)}}{c}\right)=2 \frac{v_{Q(i)}}{c} T_{P R F}
$$

where the factor $\left(c-v_{Q(i)}\right) / c$ means a Doppler shift between the frequency at which the interrogation pulses are emitted $\left(1 / T_{P R F}\right)$ and at which they are received. As $v_{Q(i)}<<c$ it can be assumed that $\left(c-v_{Q(i)}\right) / c \approx 1$. Therefore, the contact point velocity $v_{Q(i)}$ is obtained from (33) as:

$$
v_{Q(i)} \approx \frac{\Delta T_{Q(i)}}{2 T_{P R F}} c
$$

where all values in (34) are known and $\Delta T_{Q(i)}$ results from the difference of two consecutive RTOF measures. The $v_{Q(i)}$ value represents the instantaneous velocity estimation of the contact point by sending a pulse $i$. Afterwards, the train speed $v$ can be found out by averaging $N$ measurements of $v_{Q(i)}$ :

$$
v \approx \hat{v}_{(j)}=\frac{1}{N}\left(\sum_{i=j}^{i=j+N-1} v_{Q(i)}\right)
$$

Finally, the measuring process indicated on (27) only requires the knowledge of the wheel radius $R$, whose value may be smaller than the nominal one due to the wheel wear. On the other hand, the product $v T_{P R F}=\Delta x$ represents the spatial sampling interval which determines the length resolution of the irregularity.

\section{Measuring process simulation}

In practical applications, signals are interfered by electrical and structural noise leading to variations in the measured position $x_{Q(i)}$. Nevertheless the measuring method is very robust against noise due to the integration performed on (25).

Fig. 7(a) shows a simulated sequence $s(i)$ corresponding to a degenerated wheel-flat with $d$ $=0.4 \mathrm{~mm}$ in a wheel of $R=500 \mathrm{~mm}$. It has been acquired at intervals $\Delta x=v T_{P R F}=0.6$ $\mathrm{mm}$ in a rail with $c=3000 \mathrm{~m} / \mathrm{s}$. In order to test the technique performance, this sequence has been deeply contaminated with white Gaussian noise and standard deviation increasing with distance. The signal represents a typical acquisition when the Time-Gain-Compensation (TGC) function, on an ultrasound equipment, has been turned on to receive similar echo amplitudes from different distances. Interferences signify a considerable uncertainty in finding the actual echo position after every pulse $i$.

The resulting sequence $d_{k}$ obtained from the noisy signal $s(i)$ by application of (25) is shown in Fig. 7(b). It has been used a window size of $M=267$ samples, or following (26), this corresponds to a maximum wheel-flat length of $L_{\max }=320 \mathrm{~mm}$. It can be seen the filtering effect of the sum, as well as the agreement of the negative and positive peaks with the correct $d=0.4 \mathrm{~mm}$ value $\left(d_{N}=0.3898 \mathrm{~mm}, d_{P}=0.4824 \mathrm{~mm}\right)$. The averaged estimation is $d_{E}=$ $0.4361 \mathrm{~mm}$. 


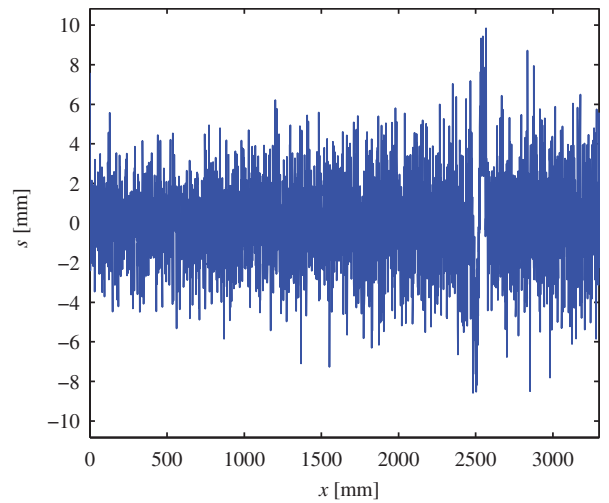

(a) Simulation of a noisy sequence $s$.

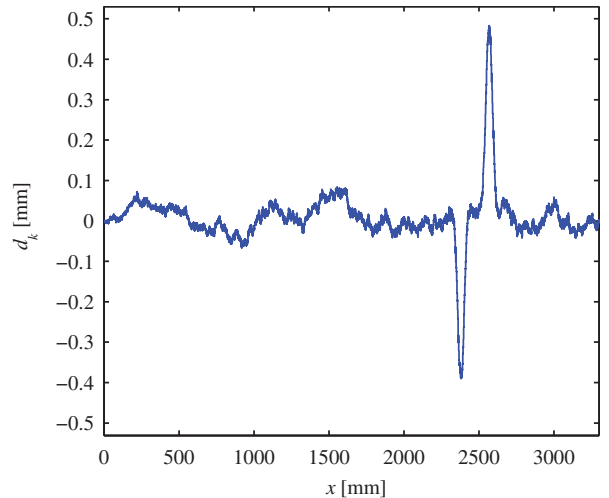

(b) Resultant $d_{k}$ sequence.

Fig. 7. Simulation parameters: $R=500 \mathrm{~mm}, c=3000 \mathrm{~m} / \mathrm{s}, d=0.4 \mathrm{~mm}$, and $\Delta x=v T_{P R F}=0.6$ $\mathrm{mm}$.

\subsection{Choosing the integration window}

In the case of a single wheel-flat, the $M$ value must be strictly chosen greater than the number of samples obtained from a cycle of $s$ in order to provide a robust estimation of $d$. However, for multiple wheel-flats which are frequently formed on modern high speed trains due to the failure of disc brakes and wheel slid prevention systems (WSP) (Grosse et al., 2002; Kawaguchi, 2006). In such case, a too large integration window may include information belonging to different flats giving wrong results.

Fig. 8(a) shows a simulated s sequence acquired at $\Delta x=0.6 \mathrm{~mm}$. The signal corresponds to three degenerated wheel-flats of $d=0.5,0.6$, and $0.4 \mathrm{~mm}$ without overlapping (separated at 157 $\mathrm{mm}$ ) in a wheel of $R=500 \mathrm{~mm}$. The resulting $d_{k}$ sequence using an integration window $M_{x}=$ $\Delta x M=150 \mathrm{~mm}$ (distance less than wheel-flats separation) provides a wrong information, since no indication is given about the largest defect of $d=0.6 \mathrm{~mm}$ (Fig. 8(b)). These effects have been originated by the size chosen for the window $M_{x}$. So when the integration is performed, $s$ values corresponding to consecutive wheel-flats are combined (note that first and last cycles, corresponding to the extreme flaws, have been correctly evaluated).

Repeating the same procedure but using a smaller window $M_{x}=75 \mathrm{~mm}$ (half of the previous case), the resulting sequence $d_{k}$ contains information according to the loss of material $d$ of each wheel-flat: $d_{N_{1}}=0.5107 \mathrm{~mm}, d_{P_{1}}=0.4859 \mathrm{~mm}, d_{N_{2}}=0.5876 \mathrm{~mm}, d_{P_{2}}=0.5485, d_{N_{3}}=0.4688 \mathrm{~mm}$, $d_{P_{3}}=0.3678 \mathrm{~mm}$ (Fig. 8(c)).

Therefore, the value of $M$ should be determined by a heuristic process trying out different integration window sizes for each obtained signal $s$. From this process, it is interesting to know the greatest estimation which is linked to the deeper wheel-flat. Moreover, from the standpoint of railway maintenance, this value indicates whether the wheel should be reprofiled or removed from service. Fig. 8(d) shows the estimations resulting of trying out different window sizes $\left(1 \leq M_{x} \leq 150 \mathrm{~mm}\right)$ on the signal shown in Fig. 8(a). The maximum value provides an estimation of the greater wheel-flat characteristic: $\max \left[d_{E}(M)\right]=0.6326$ $\mathrm{mm}$ which is a value acceptably close to the real one $\left(d_{3}=0.6 \mathrm{~mm}\right)$. 


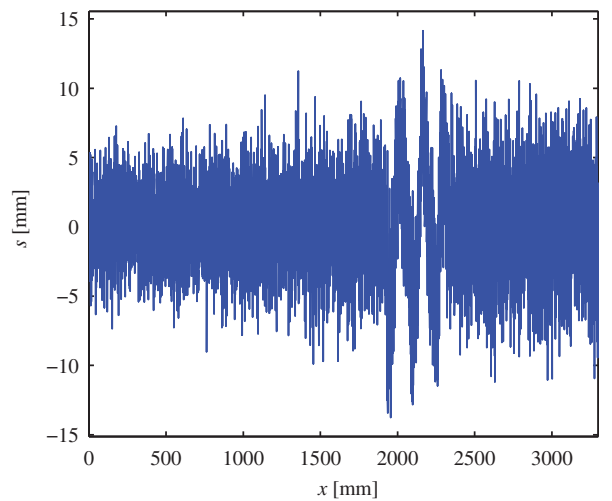

(a) Simulation of a noisy sequence $s$.

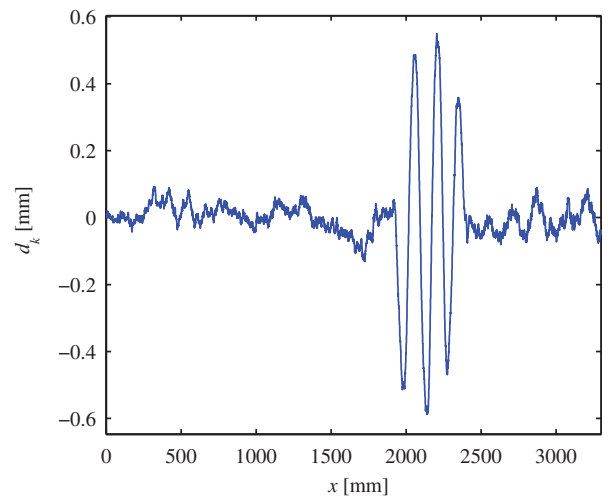

(c) Sequence $d_{k}$ corresponding to $M_{x}=75 \mathrm{~mm}$

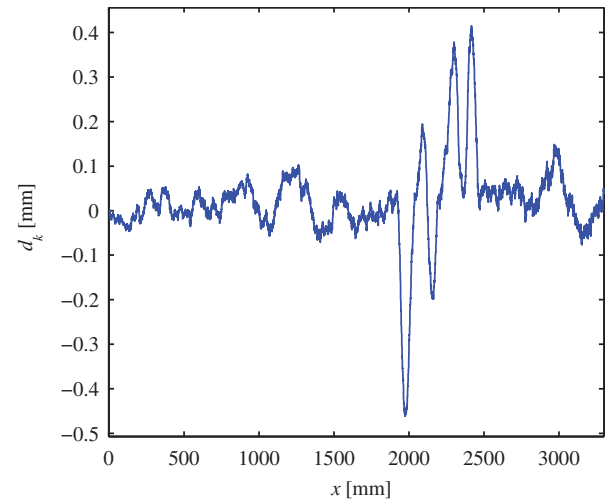

(b) Sequence $d_{k}$ using $M_{x}=157 \mathrm{~mm}$.

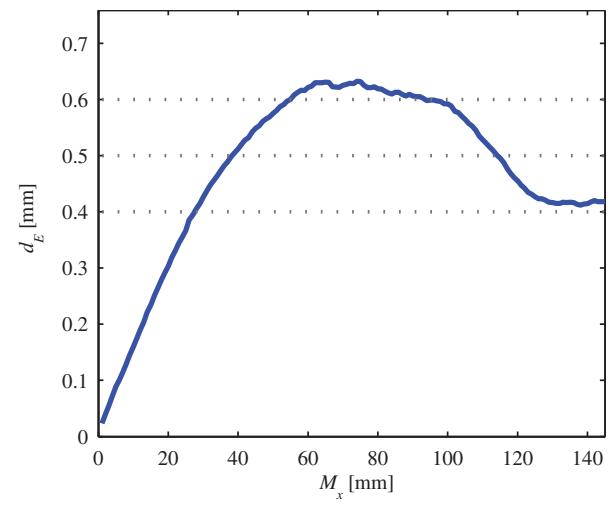

(d) $d_{E}(M)$ estimated by a multiple windows process.

Fig. 8. Simulation parameters: 3 wheel-flats without overlapping; loss of material for each wheel-flat $d=0.4,0.6,0.5 \mathrm{~mm}$; wheel radius $R=500 \mathrm{~mm}$ and $\Delta x=v T_{P R F}=0.6 \mathrm{~mm}$.

\section{Prototype inspection system}

An UltraSCOPE ${ }^{\circledR}$, Dasel Sistemas (2010), ultrasonic testing instrument was modified to support this methodology. In order to optimize bandwidth and storage requirements, the acquisition time has to be kept small. Thus, a tracking algorithm was developed and hardware implemented to make a narrow acquisition window around the rail-wheel contact point follows the contact-echo displacement (Brizuela, Ibáñez \& Fritsch, 2010).

On the other hand, the acquired signals are interfered by grain noise and many other propagation modes in the measuring rail, which cannot be removed by conventional filtering. Fortunately, this kind of noise can be partially removed because it is mostly static. Therefore, a noise cancellation procedure was also included with the tracking algorithm to avoid losing the contact echo. To this purpose, a vector formed by the difference of the absolute values between two consecutive acquisitions is obtained. Electrical noise is also reduced by applying a programmable narrow-bandpass 63-coefficients FIR filter. 


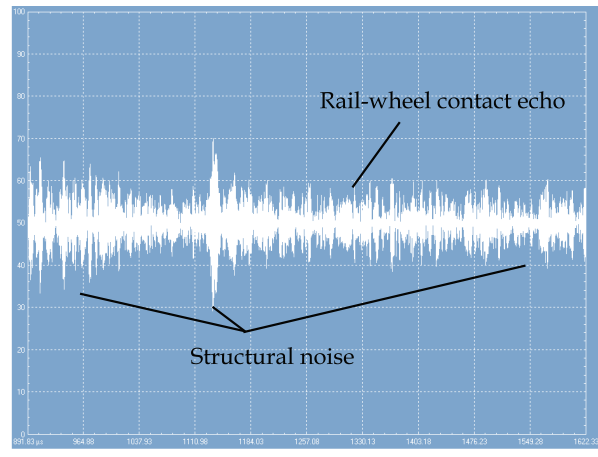

(a) Original A-scan.

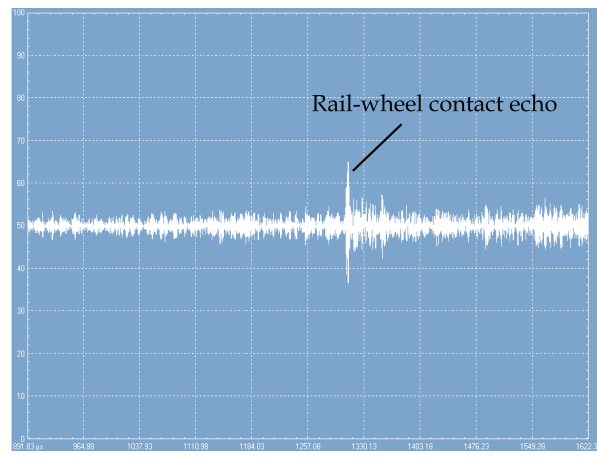

(b) Differential trace.

Fig. 9. Structural noise cancellation. In both cases the wheel is in motion near the same rail region.

Since the acquisition system operates over a differential trace, if the wheel is static or non-present, A-scans will be null excepting the electrical noise. While the wheel is in motion, A-scans will contain the information about the actual rail-wheel contact point position as a positive indication and the precedent one with negative sign. While the acquisition window is moving, the noise cancellation algorithm is performed using the samples which correspond to the same spatial point on the rail in consecutive acquisitions. Fig. 9(a) shows a high rail structural noise which masks the wheel contact-echo in the acquired signal. After structural noise removal, the wheel echo is clearly visible (Fig. 9(b)).

In addition other functions have been included, such as, Time-Gain-Compensation (TGC) to receive similar contact-point echo amplitudes from different distances; a programmable burst generator to drive transducers through a power stage (pulser). Finally, measurements are launched automatically when a wheel is detected over the rail.

The recorded echo signals are sent through a USB 2.0 interface to an evaluation computer, which looks at the position of the signal value maximum in each capture to recover the RTOF $T_{Q(i)}$ required to compute $s(i)$ and $v_{Q(i)}$ on eqs. (24) and (16).

\subsection{Test bench}

Finally, to evaluate the proposed technique performance, an experimental test bench was arranged (Fig. 10(a)). A $1 \mathrm{MHz}$ piezoelectric transducer generates Rayleigh wave pulses in a $2000 \mathrm{~mm}$ long measuring rail. A pair of wheel treads with $R=420 \mathrm{~mm}$ were used to perform the experimental work.

Two artificial wheel-flats were mechanized, one on each wheel tread. Figs. 10(b) and 10(d) show the test profiles measured with a mechanical comparator (10(c)). The maximum loss of material for the wheel-flat \#1 is $d=0.46 \mathrm{~mm}$. By application of (1) this corresponds to a new wheel-flat length of $L=39.3 \mathrm{~mm}$. A simple comparative between measured and new profiles can be also done in Fig. 10(b). Note that profile \#1 corresponds to an asymmetric partially rounded wheel-flat. 


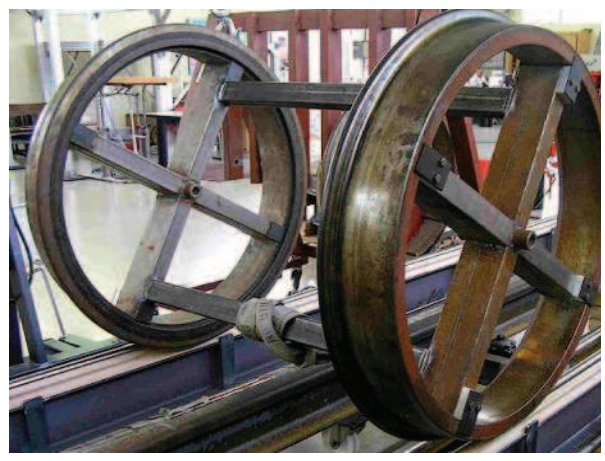

(a) Experimental test bench.

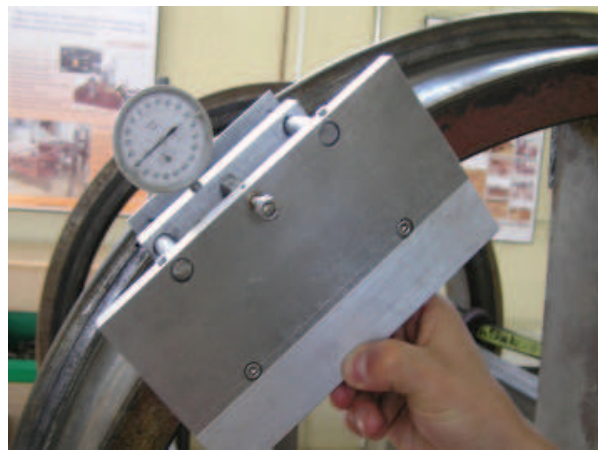

(c) Mechanical measuring instrument.

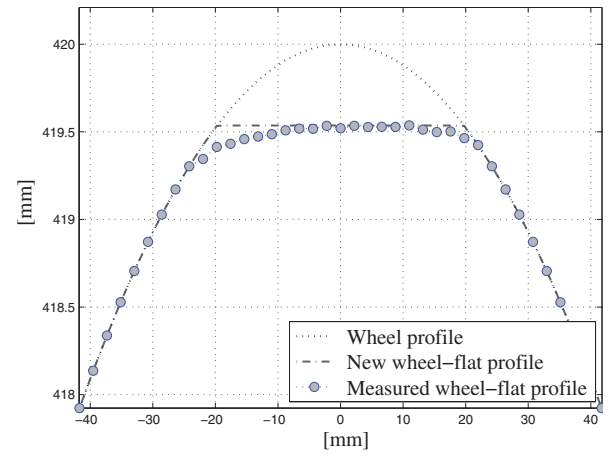

(b) Wheel-flat \#1

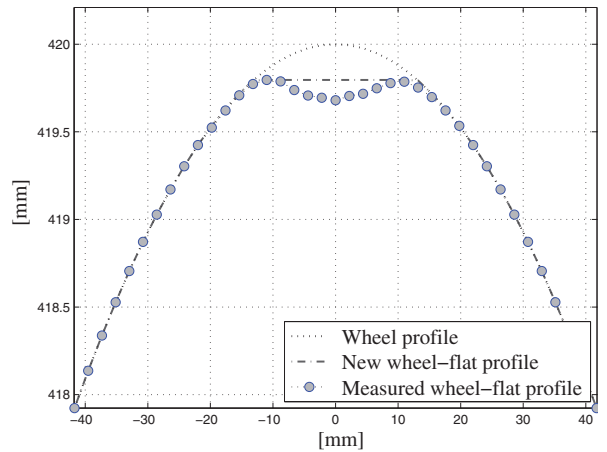

(d) Wheel-flat \#2.

Fig. 10. Prototype inspection system used for laboratory testing and artificial wheel-flat profiles.

On the other hand, the wheel-flat \#2 profile shown in Fig. 10(d), has a shape slightly concave (a cavity). This artificial flaw emulates a new wheel-flat, since it is not possible to roll over a cavity. The corresponding new wheel-flat is defined by $d=0.20 \mathrm{~mm}$ which yields to a length of $L=25.9 \mathrm{~mm}$.

\section{Experimental results}

The wheelset was moved by hand over the measuring rail, so that the speed was not quite constant. However, the rail-wheel contact position $x_{Q(i)}$ is obtained by (22) as a function of the measured RTOF $T_{Q(i)}$ and the ultrasound propagation velocity $c=2970 \mathrm{~m} / \mathrm{s}$. Consequently, the mean speed $v$ can be also estimated for every position in the rail by applying (35).

The wheel-flat \#1 was tried out under this procedure. The estimated mean speed near the flat region was $v \approx 0.45 \mathrm{~m} / \mathrm{s}$ and the spatial sampling interval was $\Delta x=v T_{P R F}=0.90 \mathrm{~mm}$. Figure 11(a) shows the contact point distance $x_{Q(i)}$ as a function of the trigger number. Note the jump in $x_{Q}$ when the wheel rolled over the wheel-flat, which is shown with more detail in Fig. 11(c). The current wheel-flat length could be obtained from this graph by observing the slope of $x_{Q}$ which changes between triggers \#820 and \#860, that is, an interval $\Delta t=80 \mathrm{~ms}$ at 


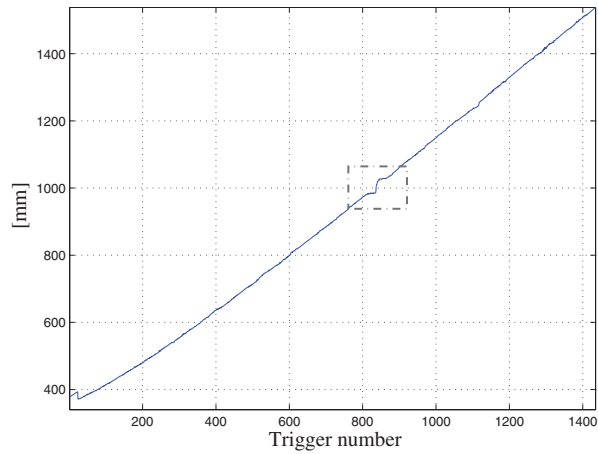

(a) Rail-wheel contact point position $x_{Q}$.

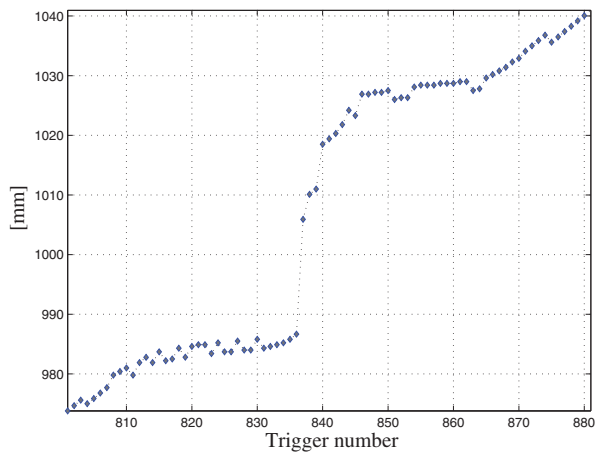

(c) Detailed view of Fig. 11(a).

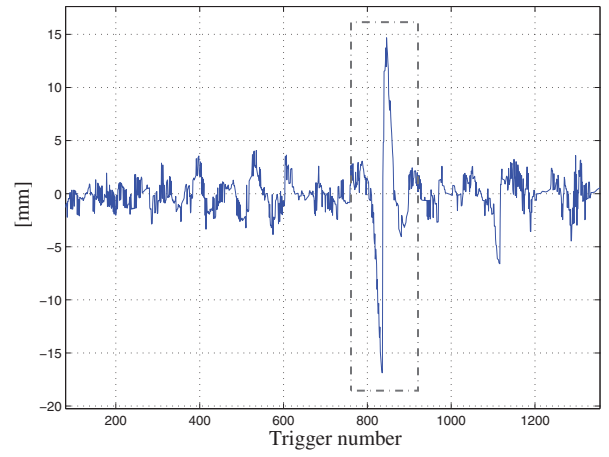

(b) Displacement signal $s$.

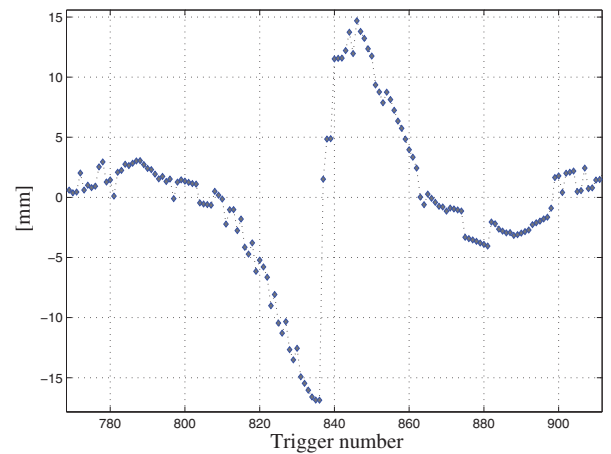

(d) Detailed view of Fig. 11(b).

Fig. 11. Wheel position obtained by measuring the RTOF $\left(T_{Q(i)}\right)$ and the relative displacement between contact point and the wheel center projection as a function of the trigger number.

$T_{P R F}=2 \mathrm{~ms}$. The wheel-flat length can be easily found out by multiplying the train speed $v$ and $\Delta t$, which yields to $L \approx 36.4 \mathrm{~mm}$. Nevertheless, this measurement method is rather imprecise, since it depends on finding the points where $x_{Q}$ changes its slope. Moreover, it gives no information about the dimensions of the original new wheel-flat (length and loss of material). Therefore, it is much better to estimate the original loss of material $d$ by applying the area calculation on $|s(i)|$.

Figure 11(b) shows the displacement $s$ around the flat region computed by (24). This sequence is contaminated by the uncertainty of locating the exact position of the echo signal due to residual noise (Fig. 11(d)). Then, (25) was applied with different window widths $(2 \leq M \leq$ 250 samples), obtaining $d_{E}(M)$ from (27).

Figure 12(a) shows the resulting estimation of $d_{E}$ as a function of $M$. It can be seen that, for $M$ values below those indicated by (26), that is, $M<16$, the estimated $d_{E}$ value shows errors. Above this figure, the estimation remains steady near the true value $(0.46 \mathrm{~mm})$, with an average $d_{E_{\text {mean }}}=0.40 \mathrm{~mm}$ and a standard deviation $\sigma_{d_{E}}=0.03 \mathrm{~mm}$, in agreement with theory. The maximum estimation is obtained when $M=27$ (or $M_{x}=24.30 \mathrm{~mm}$ ), where $d_{E}(27)$ 


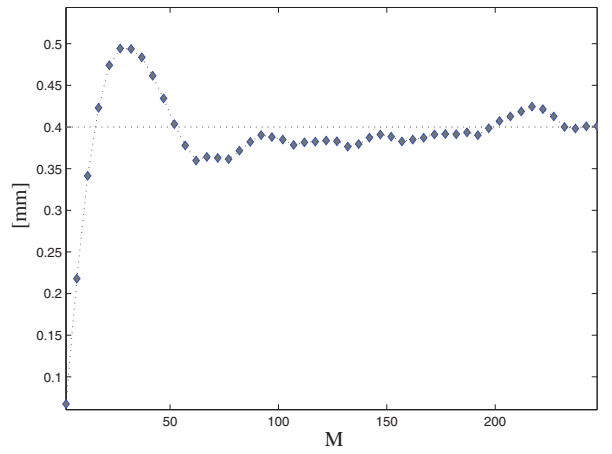

(a) Estimated loss of material $d_{E}$.

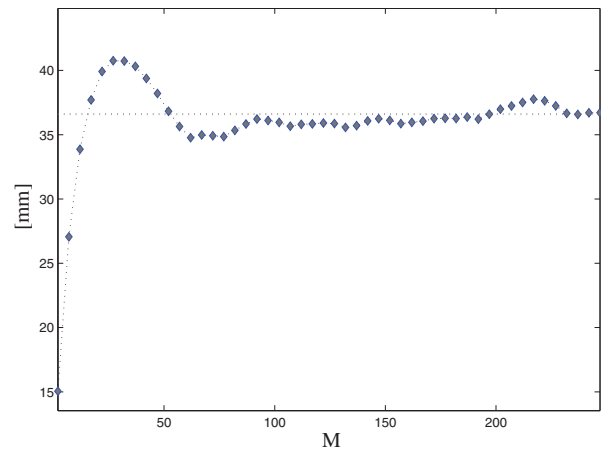

(b) Estimated new wheel-flat length $L_{E}$.

Fig. 12. Loss of material $d$ and new wheel-flat length $L_{E}$ estimations as a function of the integration window size.

\begin{tabular}{|c|c|c|c|c|c|c|c|}
\hline \multicolumn{2}{|l|}{ Parameter } & \multicolumn{3}{|c|}{ Wheel-flat\#1 } & \multicolumn{3}{|c|}{ Wheel-flat\#2 } \\
\hline Loss of material & $d[\mathrm{~mm}]$ & & 0.46 & & & 0.20 & \\
\hline Equivalent new wheel-flat length & $L[\mathrm{~mm}]$ & & 39.3 & & & 25.9 & \\
\hline Distance to transducer & $x[\mathrm{~mm}]$ & 500 & 1000 & 2000 & 700 & 900 & 1300 \\
\hline Mean speed & $v[\mathrm{~m} / \mathrm{s}]$ & 0.33 & 0.45 & 0.54 & 0.21 & 0.25 & 0.34 \\
\hline Sampling interval & $\Delta_{x}[\mathrm{~mm}]$ & 0.66 & 0.90 & 1.62 & 0.42 & 0.50 & 0.68 \\
\hline Highest estimation & $d_{E_{\max }}[\mathrm{mm}]$ & 0.45 & 0.48 & 0.56 & 0.21 & 0.20 & 0.16 \\
\hline Mean estimation & $d_{E_{\text {mean }}}[\mathrm{mm}]$ & 0.40 & 0.40 & 0.40 & 0.17 & 0.16 & 0.15 \\
\hline Estimated maximum length & $L_{E_{\max }}[\mathrm{mm}]$ & 38.90 & 40.75 & 43.72 & 27.07 & 26.16 & 23.57 \\
\hline Relative error & $\varepsilon\left(L_{E_{\max }}\right) \%$ & -1.01 & 3.68 & 11.32 & 4.51 & 0.10 & -8.99 \\
\hline Estimated average length & $L_{E_{\text {mean }}}[\mathrm{mm}]$ & 35.40 & 36.61 & 40.30 & 24.14 & 23.02 & 21.06 \\
\hline
\end{tabular}

Table 1. Wheel-flats evaluation at different distances from the transducer.

$=0.49 \mathrm{~mm}$ a value slightly higher than the measured value. In fact, it can be seen that using a large value for $M$ has little impact in the measurement of isolated wheel-flats.

Figure 12(b) shows the corresponding $L_{E}(M)$ values, using the estimations $d_{E}(M)$ and (1). The average value $L_{E_{\text {mean }}}$ is $36.61 \mathrm{~mm}$ with a standard deviation $\sigma_{L_{E}}=1.39 \mathrm{~mm}$. Note that the equivalent new wheel-flat length is $39.3 \mathrm{~mm}$. These results show a small error by defect, which are due to approaching the integral (15) by a sum (25). The maximum length estimated is $L_{E_{\max }}=40.75 \mathrm{~mm}$ and it is found at $M=27$ as well.

Following this methodology, both artificial wheel-flats were evaluated in several positions over the measuring rail in order to put them under different conditions of structural noise interference. On Table 1 the obtained results of these experiments have been summarized. Note that as the wheel speed has not been controlled, so the spatial sampling interval is different for each test.

For all cases, the lower relative error with regard to the true value is reached when the integration window extent is close to the defect length, so the estimation is maximum. The error increases when measurements are made beyond $1300 \mathrm{~mm}$ of the transducer, where 


\begin{tabular}{|c|c|c|c|c|c|c|c|c|c|c|c|}
\hline Parameter & & Wheel & l-flat \#1 & & & & & & & & \\
\hline Decimation factor & $n$ & 1 & 2 & 3 & 4 & 5 & 6 & 7 & 8 & 9 & 10 \\
\hline Mean speed & $v[\mathrm{~m} / \mathrm{s}]$ & 0.41 & 0.82 & 1.24 & 1.65 & 2.06 & 2.48 & 2.89 & 3.30 & 3.72 & 4.13 \\
\hline Spatial sampling interval & $\Delta x[\mathrm{~mm}]$ & 0.90 & 1.82 & 2.72 & 3.63 & 4.54 & 5.45 & 6.36 & 7.27 & 8.18 & 9.09 \\
\hline Window size & $M$ [samples] & 28 & 14 & 12 & 9 & 7 & 6 & 5 & 5 & 4 & 4 \\
\hline Estimated maximum length & $L_{E_{\max }}[\mathrm{mm}]$ & 40.75 & 40.09 & 40.60 & 39.47 & 39.29 & 37.72 & 37.64 & 41.43 & 42.32 & 42.93 \\
\hline Relative error & $\varepsilon\left(L_{E_{\max }}\right) \%$ & 3.69 & 2.02 & 3.32 & 0.45 & -0.01 & -4.01 & -4.22 & 5.42 & 7.68 & 9.25 \\
\hline Estimated average length & $L_{E_{\text {mean }}}[\mathrm{mm}]$ & 36.42 & 35.94 & 35.90 & 34.77 & 38.38 & 36.90 & 37.13 & 34.42 & 34.37 & 34.05 \\
\hline
\end{tabular}

Table 2. New wheel-flat estimated at different train speed using the recorded signal on Fig. 11(b).

interrogation pulse attenuation is important and structural noise interferences on the echo signal are higher, increasing the error on determining the contact point position. Nevertheless, the estimated lengths corresponding to wheel-flat \#1 have been obtained with a relative error below $12 \%$. On the other hand, detecting the wheel-flat \#2 is more difficult because it is smaller. In this case, the amplitude of the displacement signal $s$ is comparable to the residual noise. However, lengths have been estimated with an error below 9\%, which confirms experimentally the method robustness.

\subsection{Measures under different speed inspection conditions}

The technique behaviour has been also tested by making the measurements at multiple speeds (more than $3 \mathrm{~m} / \mathrm{s}$ ). The recorded displacement signals were decimated in order to increase the equivalent wheel speed. Table 2 contains the wheel-flat length estimations corresponding to the displacement signal shown in Fig. 11(b). In this case, the integration window size was bounded to $M_{x}=150 \mathrm{~mm}$. The maximum length estimated is obtained when the window size is close to the equivalent new wheel-flat length $(39.9 \mathrm{~mm})$, however fewer samples are contained on $M$ as the speed increases.

The estimated maximum lengths of the new wheel-flat at speeds up to $3 \mathrm{~m} / \mathrm{s}$ remain close to the true value with relative errors that do not exceed $5 \%$, which means an inaccuracy of 1.9 $\mathrm{mm}$. For higher train speeds the relative error increases because the spatial sampling interval will increase as well. Note also that, as the inspection speed increases the average wheel-flat lengths tend to decrease as a consequence of capturing fewer samples.

Consequently, inspection speeds that exceed a maximum value of $3 \mathrm{~m} / \mathrm{s}$ should be avoided to keep enough resolution.

\section{Conclusion}

Over this chapter, a new concept supported on a theoretical background has been disclosed to detect and measure wheel-flats in the rolling surface of railway wheels. Wheel-flats are quantified by the sum of relative displacements between the wheel-rail contact point and the wheel center projection on the rail, which yields the loss of material produced by abrasion in the original flat formation. These displacements can be obtained from ultrasonic techniques by analysing the contact point echo. Two methods based on the same measuring arrangement but different principles have been described and discussed. 
In the first proposed method based on the Doppler effect, wheel-flats can be easily distinguished. However, this technique does not allow measuring their length due to the uncertainty in the time-frequency analysis. Nevertheless, it provides a reliable wheel-flat indication, which can be very useful to give warning of failure.

On the other hand, the RTOF measuring alternative provides a high resolution which allows sizing wheel-flats. In this technique, the displacement signal $s$ is obtained from the measured RTOF and the wheel mean speed can be also estimated.

This methodology has been tested by simulation using high noise levels in signals and wheel-flats with different stages of roundness. The simulation results have proven that this technique is robust against noise and the measurement is independent of wheels wear degree and wheel-flats roundness.

An experimental test bench was built to evaluate the technique performance. The specific hardware design provides a robust support for a quantitative measurement of wheel-flats, independently of the hostile railway environment, weather conditions and wheel wear. Two artificial wheel-flats of 40 and $26 \mathrm{~mm}$ length with different wear degree were mechanized in a railway wheelset for testing. The artificial wheel-flats were placed at different positions over the measuring rail and the estimated lengths remained close to the true value with a low relative error. Thus, simulation and experimental results agree with the theoretically expected.

The inspection speed was also taken into account for the RTOF measuring method. Recorded signals were decimated in order to increase the equivalent wheel speed. The system resolution decreases as the speed increases; nevertheless estimated lengths remained close to the real value with relative errors below $5 \%$ at maximum speed $(3 \mathrm{~m} / \mathrm{s})$.

The proposed methodology, as being a dynamic technique without moving parts but with a well-characterized and stable measuring arrangement, is suitable for the railway industry. The system allows a periodical wheel inspection, improving reliability, availability and effective operations of the railway system, guaranteeing high safety standards. Moreover, it allows reducing the maintenance costs. The inspection procedure can be performed while the train is getting into a repair shop reducing the time spent in maintenance scheduled tasks. As a result, frequently inspections can allow to follow-up the wheel wear history by uploading the information into a database and optimizing wheels service.

\section{References}

Baeza, L., Roda, A., Carballeira, J. \& Giner, E. (2006). Railway train-track dynamics for wheelflats with improved contact models, Nonlinear Dynamics 45: 385 - 397.

Belotti, V., Crenna, F., Michelini, R. \& Rossi, G. (2006). Wheel-flat diagnostic tool via wavelet transform, Mechanical Systems and Signal Processing 20(8): 1953-1966.

Bray, D., Dalvi, N. \& Finch, R. (1973). Ultrasonic flaw detection in model railway wheels, Ultrasonics 11(2): 66-72.

Brizuela, J., Ibáñez, A. \& Fritsch, C. (2010). NDE system for railway wheel inspection in a standard FPGA, Journal of Systems Architecture 56: 616 - 622.

Brizuela, J., Ibáñez, A., Nevado, P. \& Fritsch, C. (2010). Flaw detector for railways wheels by doppler effect, Physics Procedia 3(1): $811-817$.

Dasel Sistemas (2010). Ultrasound technology. viewed March 22, 2012, http:/ / www.daselsistemas.com. 
Fan, H. \& Jia, H. (2008). Study on automatic testing of treads of running railroad wheels, Proceedings of the $17^{\text {th }}$ World Conference on Nondestructive Testing, $17^{\text {th }}$ WCNDT 2008, Shangai, China.

Feng, Q., Cui, J., Zhao, Y., Pi, Y. \& Teng, Y. (2000). A dynamic and quantitative method for measuring wheel flats and abrasion of trains, $15^{\text {th }}$ World Congress on NDT, Rome, Italy.

Grosse, M., Ceretti, M. \& Ottlinger, P. (2002). Distribution of radial strain in a disc-braked railway wheel measured by neutron diffraction, Applied Physics A: Materials Science and Processing 74: 1400 ? 1402.

Gutauskas, P. (1992). Railroad flat wheel detectors. US Patent No. 5133521.

He, P., You, Z. \& Teng, S. (2005). Fast algorithm of flat sliding detection in flat wheel detecting system, Instrumentation and Measurement Technology Conference, IMTC 2005, Ottawa, Canada.

Ibáñez, A., Parrilla, M., Fritsch, C. \& Giacchetta, R. (2005). Inspección mediante ultrasonidos de ruedas de tren en operaciones de mantenimiento, $V$ CORENDE Proceedings, Neuquén, Argentina.

Kappes, W., Kröning, M., Rockstroh, B., Salzburger, H.-J. \& Walte, F. (2000). Non-destructive testing of wheel-sets as a contribution to safety of rail traffic, CORENDE 2000 Proceedings, Mar del Plata, Argentina.

Kawaguchi, K. (2006). Development of a wsp system for freight trains, $7^{\text {th }}$ World Congress on Railway Research (WCRR2006 Proceedings)., Montreal, Canada.

Kumagai, N., Ishikawa, H., Haga, K., Kigawa, T. \& Nagase, K. (1991). Factors of wheels flats occurrence and preventive measures, Wear 144: 277 - 287.

Madejski, J. (2006). Automatic detection of flats on the rolling stock wheels, Journal of Achievements in Materials and Manufacturing Engineering 16(1-2): 160-163.

Pohl, R., Erhard, A., Montag, H.-J., Thomas, H.-M. \& Wüstenberg, H. (2004). NDT techniques for railroad wheel and gauge corner inspection, NDTEE International 37: 89 - 94.

Salient Systems, Inc. (2010). Intelligent track solutions. viewed March 22, 2012, http:/ / www.salientsystems.com.

Salzburger, H. J., Wang, L. \& Gao, X. (2008). In-motion ultrasonic testing of the tread of high-speed railway wheels using the inspection system AUROPA III, $17^{\text {th }}$ World Conference on NDT, Shangai, China.

Snyder, T. \& Stone, D. H. (2003). Wheel flat and out-of-round formation and growth, Proc. 2003 IEEE/ASME Joint Rail Conf., Chicago, Illinois, pp. 143 - 148.

Stratman, B., Liu, Y. \& Mahadevan, S. (2007). Structural health monitoring of rail road wheels using impact load detectors, Journal of Failure Analysis and Prevention 7(3): 218 - 225.

Vyas, N. S. \& Gupta, A. K. (2006). Modeling rail wheel-flat dynamics, Engineering Asset Management, Springer London, pp. $1222-1231$.

Wu, T. X. \& Thompson, D. J. (2002). A hybrid model for the noise generation due to railway wheel flats, Journal of Sound and Vibration 251(1): 115 - 139.

Zakharov, S. M. \& Goryacheva, I. G. (2005). Rolling contact fatigue defects in freight car wheels, Wear 258: 1142 - 1147.

Zerbst, U., Mädler, K. \& Hintze, H. (2005). Fracture mechanics in railway applications - an overview, Engineering Fracture Mechanics 72(2): 163-194. 


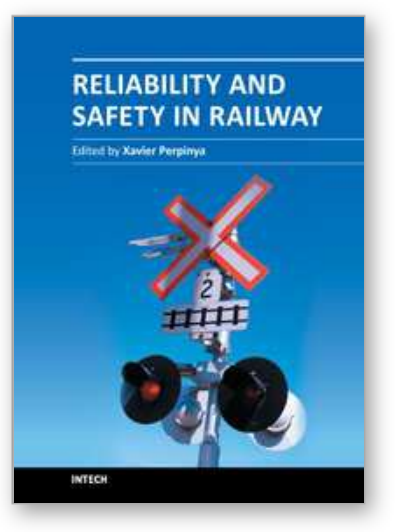

\author{
Reliability and Safety in Railway \\ Edited by Dr. Xavier Perpinya
}

ISBN 978-953-51-0451-3

Hard cover, 418 pages

Publisher InTech

Published online 30, March, 2012

Published in print edition March, 2012

In railway applications, performance studies are fundamental to increase the lifetime of railway systems. One of their main goals is verifying whether their working conditions are reliable and safety. This task not only takes into account the analysis of the whole traction chain, but also requires ensuring that the railway infrastructure is properly working. Therefore, several tests for detecting any dysfunctions on their proper operation have been developed. This book covers this topic, introducing the reader to railway traction fundamentals, providing some ideas on safety and reliability issues, and experimental approaches to detect any of these dysfunctions. The objective of the book is to serve as a valuable reference for students, educators, scientists, faculty members, researchers, and engineers.

\title{
How to reference
}

In order to correctly reference this scholarly work, feel free to copy and paste the following:

Jose Brizuela, Carlos Fritsch and Alberto Ibáñez (2012). New Ultrasonic Techniques for Detecting and Quantifying Railway Wheel-Flats, Reliability and Safety in Railway, Dr. Xavier Perpinya (Ed.), ISBN: 978-95351-0451-3, InTech, Available from: http://www.intechopen.com/books/reliability-and-safety-inrailway/ultrasonic-dynamic-technique-for-detecting-and-quantifying-railway-wheel-flats-

\section{INTECH}

open science | open minds

\section{InTech Europe}

University Campus STeP Ri

Slavka Krautzeka 83/A

51000 Rijeka, Croatia

Phone: +385 (51) 770447

Fax: +385 (51) 686166

www.intechopen.com

\section{InTech China}

Unit 405, Office Block, Hotel Equatorial Shanghai

No.65, Yan An Road (West), Shanghai, 200040, China

中国上海市延安西路65号上海国际贵都大饭店办公楼405单元

Phone: +86-21-62489820

Fax: $+86-21-62489821$ 
(C) 2012 The Author(s). Licensee IntechOpen. This is an open access article distributed under the terms of the Creative Commons Attribution 3.0 License, which permits unrestricted use, distribution, and reproduction in any medium, provided the original work is properly cited. 